\title{
Effects of Chinese and English Word Segmentation on Reading Speed
}

\author{
Yanping Ling ${ }^{1}$ Dixiu Liu ${ }^{1, *}$ \\ ${ }^{I}$ Department of Psychology, Gannan Medical University, Ganzhou, Jiangxi 341000, China \\ *Corresponding author.Email:513718686@qq.com
}

\begin{abstract}
62 college students who passed College English Test Band 4 (CET-4) were selected as the participants in this study. Through two experiments, the researchers used the E-prime online experimental platform to explore the effect of word segmentation on the reading rate of Chinese and English sentences. The independent variables were the reading material and the segmentation method, and the dependent variables were the subjects' reading time and reading accuracy. The researchers found that the reading time of English sentences was significantly longer than that of Chinese; and the reading time of inter-word space was the shortest, but the reading time in non-word space was the longest. When the participants were reading Chinese sentences, there was no significant difference in reading response under the three conditions. When the participants were reading English sentences, there was a significant difference in reading response under the three conditions. It showed that the type of reading material had an impact on the reading of college students, and the segmentation level was the main factor affecting reading. Moreover, words are an important processing unit in Chinese reading.
\end{abstract}

Keywords: Chinese, English, Word segmentation, Reading speed.

\section{INTRODUCTION}

The basic writing unit of Chinese is Chinese characters [1]. In standard Chinese texts, each Chinese character in a sentence is next to each other. Except for some sentences that need to be paused with punctuation marks such as pauses, there is no other prominent word boundary information. The uniqueness of Chinese is that the unit of writing does not correspond to the unit of meaning. The unit of writing in Chinese is a Chinese character, but sometimes a single Chinese character is meaningless [2]. The smallest unit of meaning in Chinese is a word composed of multiple characters. Therefore, it is necessary to segment the words correctly before the researchers can better understand the meaning of the sentence. Word segmentation refers to the division of text into "words" through a certain reading mechanism. Word segmentation is the primary link in vocabulary processing and plays a very important role in reading [3]; otherwise, it will cause ambiguity in the sentence and cause understanding obstacles. The writing of Chinese characters is completely different from that of English: when writing Chinese characters, clear boundary information between words is not added, while when writing in English, words are separated by spaces. Sometimes, it is difficult for native Chinese speakers to segment words, but in Chinese culture, they will grow up and gradually form a rich reading experience. Therefore, despite the lack of word boundary information in standard Chinese texts, general readers can correct themselves in time when reading back or after careful thinking [4]. However, for foreign students whose mother tongue is a Pinyin text background and their Chinese proficiency is at an intermediate or lower level, they are accustomed to reading text forms with word boundary information. For this reason, they will face barriers in word segmentation when reading Chinese texts. They will split the words in a sentence into several unrelated words, or group adjacent words into non-words, which will further interfere with their reading [2]. But in Pinyin text, English words are separated by spaces, and readers can use space clues to segment words when reading. Native Chinese speakers do not encounter much trouble when reading texts without word boundary information, and the difference in writing style 
between Chinese and English leads to differences in readers' reading comprehension rate and reaction time, but Chinese readers and English readers read words at the same speed per minute [5]. Some studies have explored the effect of space between words on reading rate in text reading, but each research has different conclusions. There are disagreements on whether word segmentation in Chinese reading affects sentence reading comprehension. Regarding the impact of word segmentation in Chinese reading on reading, the results of previous studies have three categories: promotion, hindrance, and non-influence.

At present, it is quite common to explore the influence of different Chinese segmentation methods on reading. Foreign students and other Chinese beginners or Chinese native speakers are selected as the research participants. The main test sets different blank presentation conditions to investigate its influence on reading performance. For example, Winskel et al. selected Thai-English bilinguals as the research participants to explore the effect of inserting space in Thai text without space on their reading of Thai text [6]. They found that adding blank space could help readers to identify words faster, but the readers' eye movement control and word segmentation were not affected [7]. Bai and et al. took American college students as the research participants whose native language is English and whose Chinese proficiency is low. Their results showed that when foreign students were reading Chinese, word segmentation could promote Chinese reading for Chinese beginners [8]. Shen et al. also used the same experimental conditions as Bai et al., and selected students from the United States, South Korea, Japan, and Thailand as the research participants. They found that the text characteristics of these four languages were different, and the final result found that regardless of the participants' native language whether there were spaces between words and the conditions of spaces between words could effectively improve the reading performance of the participants [9]. Yu et al. screened 21 valid literatures examining the role of inter-word space in Chinese text reading. The meta-analysis method was adopted for analysis, and analyzed them using meta-analysis methods. The analysis results showed that spaces between words effectively improved the reading performance of foreign students in Chinese texts [10]. In some experiments with foreign students as the research participants, the addition of inter-word spaces could promote the Chinese reading of the participants, regardless of whether there were inter-word spaces in the texts of the subjects' native language [4]. Bai et al. further investigated the effect of inter-word space on the reading of the elderly under the same experimental conditions as Bai's. The results showed that the elderly had difficulty reading non-word space conditionals and inter-word space conditionals, but the elderly had no difference when reading interword space text as normal text, and the inter-word space text was beneficial to word recognition of the elderly [11]. A study conducted by Liu et al. found that it took longer time for readers to read the conditional text with inter-word space than the text without space. Therefore, it was concluded that inter-word space was not conducive to Chinese reading [12]. However, in the Chinese text without space itself, adding space between words in the sentence is conducive to reading comprehension. However, in English texts they have word boundaries, and deleting the spaces between words will hinder readers from reading [8][13].

However, some studies indicate that the space between words in the text has no effect on reading rate. Bai et al. used college students as the research participants to study the role of spaces between words in Chinese reading and the importance of words in Chinese reading. The results showed that the reading efficiency of the participants decreased under the conditions of inter-word space and non inter-word space. The condition of inter-word space neither hindered nor promoted the subjects' reading [14]. Although the analysis of inter-word space provided the boundary information for the participants, the unfamiliarity caused interference when the subjects read inter-word space text. The promoting effect of inter-word space may be balanced with the hindrance caused by the unfamiliarity of the text, so the promoting effect of inter-word space on reading was not reflected in the experimental data at last [4].

Some experiments also found that word segmentation marking word boundaries did not promote sentence reading. Gao selected Japanese and Korean learners as her participants. It was found that no matter elementary learners, intermediate learners or Chinese native speakers read text with increased space between words; these readers did not improve their reading speed [15]. Liu et al. selected Chinese text with space between two consecutive words as the experimental material. The experimental group read the text with word boundaries, while the control group read the text without word boundaries. The results showed that the experimental group was slower than the control 
group in reading [12]. This showed that artificially adding spaces between words does not promote word recognition and further affects Chinese reading; the subjects had been exposed to Chinese since childhood, and native speakers were accustomed to reading standard Chinese texts without Spaces. However, the artificial addition of Spaces between words in sentences broke the original visual cues, which was contrary to the eye movement habits of the participants in normal reading. Another explanation was that the addition of spaces pushes the characters out of sight, causing the participants to have difficulty reading and eventually slow down.

Not only does the presentation of word segmentation have different effects on reading, but the language characteristics also affect the effect of the space factor, the study of Bai et al. found that Indian-English bilinguals could read in English under two conditions: no space delimiter and gray condition and no space condition, and the speed significantly dropped faster than that in Hindi, which showed that language characteristics had a great influence on whether the space factor plays a role in reading [16]. In English, studies have found that the spaces between words in sentence played an important role in word recognition, which could improve the sentence processing rate. Removing or shade spaces between words would severely hamper the normal reading process, and thus result in a decline in their reading efficiency about $30 \%$ to $50 \%$ [6][17]. Moreover, in the Pinyin writing system of English, words are the unity of writing units and meaning units. Reading performance would be reduced by $30 \%$ to $50 \%$ if blank word boundary markers were removed between English words [18][19][20][21].

The existing research results were intent to verify the influence of different way of segmentation of in Chinese reading, and the influence on bilingual had provided certain experimental support, but the influence of segmentation method on Chinese and English reading remains unclear. Is there any difference in the influence of the middle term in both Chinese and English sentence comprehension? In this study, the researchers intend to explore the influence of different segmentation methods on reading efficiency in Chinese and English reading materials, aiming to make a breakthrough in theoretical perspective.

\section{MATERIALS AND METHODS}

\subsection{Material Evaluation Test}

\subsubsection{Participants}

10 college students majoring in English who did not participate in the formal experiment were randomly selected ( 5 boys and 5 girls). In addition, 2 Chinese teachers from Guangzhou 89 Middle School and two college English teachers participated in the evaluation of experimental materials.

\subsubsection{Experimental Materials}

\subsubsection{Preparation of Materials}

The researchers Chose 200 Chinese sentences and 200 English sentences based on the following principles: 1) Chinese example sentences were composed of common words of Putonghua Proficiency Test (PSC) and compiled by the main examiner; 2) the English example sentences were selected from the CET-4 market commonly used bibliography "Scar English", which has certain reliability and authority; 3) the materials of the Chinese example sentences were derived from common words in the Putonghua Test (PSC) syllabus; 4) the English example sentences were derived from common words and high-frequency words that meet the requirements of CET-4, which ensured that most of the example sentences in the experimental test were easy to understand and would not affect the reading of the subjects because of the difficulty in understanding the example sentences.

\subsubsection{Material Evaluation}

4 teachers and 10 college students who did not participate in the formal experiment took part in material evaluation. They carefully read 100 sample sentences in both Chinese and English, and then marked the sentences that students might not know or have difficulties in understanding, and evaluated the sentence smoothness and rationality on a 7 point scale, so as to make the sentences consistent in length, difficulty in understanding and smoothness as far as possible, and to exclude the influence of irrelevant variables on the research results influence [16]. 


\subsubsection{Sentence Screening}

A seven-point scoring was used to eliminate sentences with an average score of less than 4 for the same sentence by all raters. Sentences with an average score of $\geq 4$ are selected as the formal experimental sentences, and their rationality and commensurability are relatively reliable. Finally, 120 formal experimental sentences that can meet the experimental requirements are formed in terms of sentence length, commensurability and rationality.

\subsection{Experiment}

\subsubsection{Participants}

A random selection of 62 college students who have passed the National College Student Level 4 Examination (excludes the participants who have done the related evaluation test and volunteered to participate in the experiment before). Their average age was 22.5 years, and the selected participants had normal intellectual development, no dyslexia, normal vision, and right-handedness. The subjects' usual habit of reading text is the same.

\subsubsection{Stimuli}

For all experimental sentences, 120 sentences from the previous word segmentation test and 20 sentences with an average value close to 4 in the evaluation test were selected as exercise sentences. According to the modern Chinese dictionary and the Chinese character application level issued by the National Language Work Committee of the Ministry of Education of the People's Republic of China And the test outline to segment Chinese sentences. According to the CET-4 syllabus, English sentences are segmented, and sentences that are ambiguous after segmentation are deleted. This experiment is divided into practice experiment and formal experiment successively; the formal experiment has a set of Chinese materials and English materials, each group has 60 formal experimental sentences, each condition includes 20 sentences, there are 12 sentences with questions in the three sentence presentation modes of the Chinese experiment and the English experiment.

\subsubsection{Instruments}

The experimental equipment prepares a laptop computer, E-prime design experiment program, and uses the online Thues psychological experiment system provided by Beijing Hunyuan Times Technology Co., Ltd. to test, and the stimulus presentation and timing are accurate to milliseconds.

\subsubsection{Experimental Design and Procedure}

The experiment used 2 (reading material: Chinese or English) $\times 3$ (text word segmentation method: spaces between characters or words space, or non-word space) in-subject experimental design.

The experimental materials were presented on the participants' laptops. The Chinese font was boldface and 16 point, and the English font was of the same color and size. The experiment was counterbalanced by ABBA. The experiment was conducted alone at home, and the participants were asked to read before the formal experiment. 10 sentence exercises (Taking Chinese as an example, two sentences were followed by question sentences, and the subjects were asked to make a judgment by pressing a key: "A" for correct, "L" for wrong. The remaining eight sentences had no questions, and the answers were yes or no, half of which were balanced). These practice trials mainly let participants to get familiar with the experiment process. After login in Thues psychological experiment system, participants clicked "run", and wait for a few seconds after the instructions first appear on the screen interface. The instructions were shown for participants who would press key "F" on the keyboard to start practice. The screen would appear at first a point " + " for 100 milliseconds, after that an experimental sentence presented immediately, and participants were asked to read the sentence until they pressed the key "Space". Computer would record the response time of the subjects. After reading the sentence, questions related to the sentence will appear at random, and the subject needs to make judgment: press key "A" if it conforms to the previous sentence, and press key "L" if it does not. (See "Figure 1").

The experimental procedures of the formal experiment and the practice experiment were the same. The number of yes or no answers for the questions in the two groups of experimental materials was the same. Six questions needed to be judged as "yes" and six questions needed to be judged as "no". 


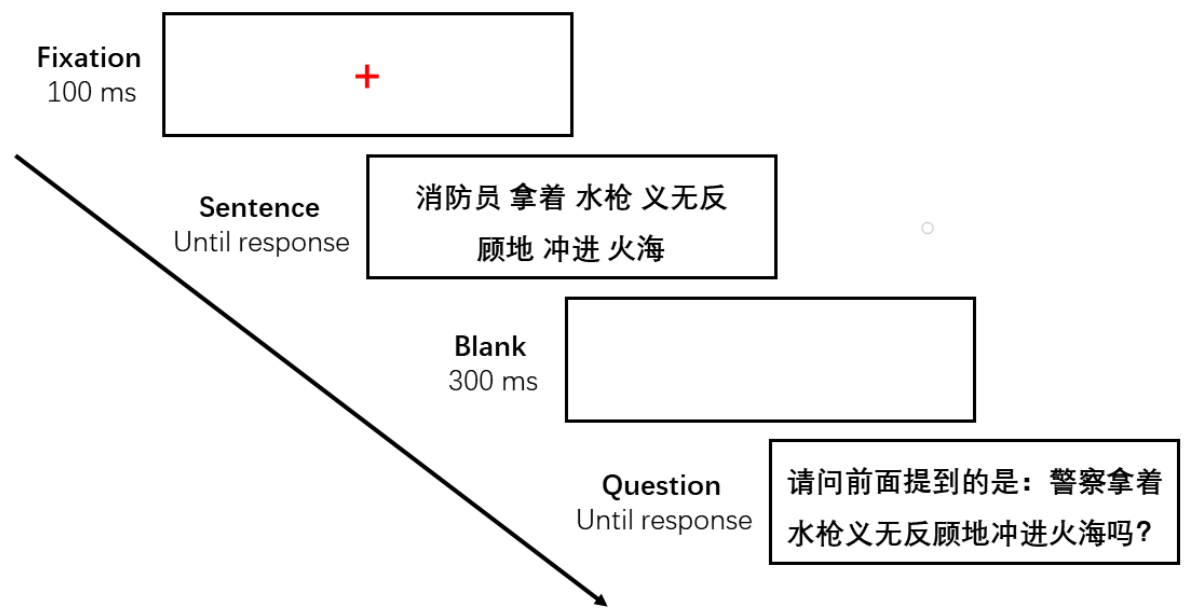

Figure 1 Task sequence and timing of a sample trial, in which the sentence is in Chinese.

\subsubsection{Data Analysis}

The data collected by E-Prime2.0 in E-studio was merged by E-Merge and then exported. Extreme data was first eliminated. In this study, extreme data that subjects did not understand sentences due to quick pressing of keys was first eliminated according to the index of "ACC (accuracy) and RT (reaction time) of questions". In this study, the reaction time of the experimental sentence was selected as the reading index parameter for the final analysis and summary of the data, and the accuracy and reaction time of the experimental sentence question sentence were used as a reference. After preliminarily collating and statistical calculation of these data using Excel,
SPSS21.0 statistical software is used to perform two-factor repeated measurement variance statistical analysis of the data.

\section{RESULTS}

\subsection{The Overall Reading Time of Chinese and English Sentences Under Different Segmentation Levels and Different Reading Materials}

Under different reading materials, different segmentation methods were used as independent variables, and the response of the subjects was the dependent variable for descriptive statistical analysis. The results were shown in "Table 1".

Table 1. Comparison of the effects of reading materials and segmentation on sentence reading response time (M $\pm \mathrm{SD}$ )

\begin{tabular}{lll}
\hline \multicolumn{1}{c}{ Conditions (i.e. segmentation levels) } & Chinese (M \pm SD, $m s)$ & English (M $\pm S D, m s)$ \\
\hline Blank space between characters (letters) & $1993 \pm 622.1$ & $4435 \pm 1335.8$ \\
Space between words & $1905 \pm 707.9$ & $3852.9 \pm 1512.1$ \\
Blank space between non-single words & $2073 \pm 744.2$ & $5497.4 \pm 2089.3$ \\
\hline
\end{tabular}

Note: The unit of RT was $m s$

"Table 1" showed the mean value (M) and standard deviation (SD) of the response time of the participants at different segmentation levels when reading Chinese and English sentences. It showed that when reading English sentences, the response time (RT) of the participants at three segmentation levels was significantly higher than that of Chinese sentences. In English non-inter-word space condition, the average RT was the longest, while the shortest RT was that in the inter-word space sentence condition when reading Chinese sentence. Regardless of whether it was in Chinese or English, under the three blank presentation conditions, the order of response time was: non-word space RT > character (letter) space RT > word space RT. The most obvious fluctuation was in English sentences with non-word spaces presenting conditions; the order of English volatility was: non-word spaces 
presenting conditional sentences $>$ inter-word spaces presenting conditional sentences > letter spaces presenting conditional sentences. The least fluctuation was the Chinese sentence under the condition of the space between words, and the difference between individuals was very small. The order of volatility was as follows: non-word spaces present conditional sentences > inter-word spaces present conditional sentences> inter-character spaces present conditional sentences. This was inconsistent with the volatility of the English blanks.

\subsection{The Reading Accuracy of Chinese and English Sentences Under Different Segmentation Levels}

It can be seen from "Table 1" and "Table 2" that when the participants were reading Chinese sentence materials, the inter-word space condition showed the fastest response time and the lowest accuracy rate in the sentence. The reading accuracy rate under each condition was as follows: non-word space ACC > Space between words ACC> Space between words ACC; when the participants read each presented sentence in English, the reading accuracy rate was the highest when the space between letters were presented. The reading accuracy of each conditional sentence was in order: space between letters> space between non-words> between words Spaces.

Table 2. The reading accuracy of Chinese and English sentences at different segmentation levels

\begin{tabular}{|c|c|c|}
\hline Conditions (i.e. segmentation levels) & Chinese $(\mathrm{M} \pm \mathrm{SD}, \%)$ & English (M $\pm S D, \%)$ \\
\hline Space between characters (letters) & 90.42 & 91.25 \\
\hline Space between words & 76.25 & 66.7 \\
\hline Blank space between non-single words & 94.58 & 84.6 \\
\hline
\end{tabular}

After further multiple comparisons, it was found that the participant's reading response under the condition of Chinese and English word segmentation: non-word space conditional present sentence> character space conditional present sentence> inter-word space conditional present sentence; The Chinese reading time difference under the three segmentation methods was small. The total sentence reading time under the condition of inter-word spaces was the shortest, and the difference was not significant with the conditions of inter-word spaces and non-inter-word spaces. However, English was completely different. The participants have the longest average response time when reading English sentences without spaces between words. This kind of sentence presentation method that completely breaks the English pinyin text makes word recognition more difficult, which brought great difficulties to the participants in reading sentences, and the accuracy of related reading comprehension would also be reduced.

The results of $2 \times 3$ two-factor repeated measurement ANOVA showed that the main effect of reading material type was very significant, $F(1,59)=208.472, \quad p=0.000, \quad p<0.001, \quad \eta^{2}=0.779$. For the type of reading materials, English reading response time was significantly higher than Chinese reading response time. Moreover, the main effect of segmentation level was also significant, $F(2,118)=68.745, \quad p=0.000, \quad p<0.001, \quad \eta^{2}=0.538$. Further multiple comparisons found that the reading speed of inter-word space was the fastest, followed by inter-word space, and non-word space was the slowest. The interaction effect of reading material type $\times$ segmentation method was significant, $F(2,118)=43.737, \quad p=0.000, \quad p<0.001$, $\eta^{2}=0.426$. Reading in English and Chinese sentence for 3 different slit mode had carried on the simple effect, simple effect test result indicated that the participants in reading Chinese sentences, there was no significant difference in reading response under the conditions of inter-character spaces, inter-word spaces, and non-word spaces. There was almost no difference in the reading speed of participants under the three segmentation levels. The specific performance is that under the spaces between characters and words, there was no significant difference in the participants' reading responses, $t(59)=1.732, p>0.05$. Under the spaces between words and non-words, the differences in reading responses were not significant, $t(59)=-1.514$, $p>0.05$. Compared with non-word spaces, the reading responses were not significant, $t(59)=$ $3.115, p<0.05$. There were significant differences in the participants' reading responses. However, when the participants read English sentences, there was a significant difference in reading response between 
the three conditions of space between letters, spaces between words, and non-word spaces, which were manifested in the conditions of space between letters and spaces between words, $t(59)=4.456$, $p<0.05$. The difference between the two was significant. In the conditional sentence with spaces between letters and non-words, $t(59)=-6.053$, $p<0.05$, and the difference between the two was also significant. Under the conditional sentences with spaces between words and non-words, $t(59)=$ $11.846, p<0.05$, there was a significant difference between the two.

\section{DISCUSSION}

Not Spaces between words in English sentences, this completely break the presentation of this kind of alphabet writing in English sentence; word recognition becomes more difficult, and also will reduce the accuracy of reading comprehension, the segmentation of space between letters can help English learners to learn English. This kind of artificial division of physical boundaries between a word requires readers to integrate the letters with spaces with greater information density in a unit space into a word with complete meaning, and the amount of natural processing information will increase. It takes a certain amount of cognitive resources and processing time for readers, and the average saccade distance of readers is shorter, which will reduce the reading speed of English learners to a certain extent. However, from the experimental results, the condition of the space between letters shows that the ACC of sentence reading comprehension is the highest. For English materials, the best way to learn is to use normal spaces between words. Especially for those of us who are new to English, the researchers need to understand sentences faster, especially when reading an English article. The sentence presentation method can achieve a better learning effect. For Chinese materials, the reading rate of subjects under various conditions will not be too affected. This is similar to the results of Bai et al.'s research. The reason why word segmentation did not promote reading was that the promoting effect of word segmentation on reading would be offset by the hindrance effect of the subjects' unfamiliarity with blank text [14].

To a large extent, this may be attributed to the fact that for Chinese native speakers, readers can easily understand the sentence even under the nonword space, based on their familiarity with Chinese, And under the conditional sentences presented with three spaces, the accuracy of answering related reading comprehension questions is the highest. But, in contrast, the condition of three kinds of segmentation Spaces between words reading speed is the fastest, so for some Chinese beginners, because their grammatical knowledge, experience and understanding of Chinese history and culture, especially for some easy to cause ambiguity and reading comprehension obstacles of idioms between word segmentation can be done, it will help Chinese beginners learn Chinese faster. The results of this study showed that the space condition between Chinese words decreased the reading time under the sentence, but the insertion of space between words affected the correct rate of reading comprehension. This is consistent with the conclusion drawn by Hsu et al. Hsu allows participants to read the traditional way of arranging the text and inserting the word boundary text; the research results found that the space between words does not improve the accuracy of sentence understanding, but can shorten the reading time of participants(Hsu et al., 2000). Furthermore, for Chinese reading materials, compared with the other two types of space-present conditional sentences, it balances the length and difficulty of the sentence. This experiment wants to investigate the effect of segmentation on the reading rate of the participants. Therefore, the participants will not encounter much dyslexia when reading sentences with spaces between letters and non-word spaces participants have a speed-accuracy balance when reading interword spaces. Accuracy must be sacrificed while improving reading speed. Therefore, there will be a reduction in reading time and accuracy of reading comprehension. when participants reading English material, and the Spaces between the letters of conditional sentence accuracy is higher than the Spaces between words, the reason is that the participants are not native English speakers, the Spaces between words completely broken English sentences of this kind of phonetic writing way, takes the participants more reading time, reduce relative accuracy of reading comprehension.

Under the three segmentation levels, the decrease of the English reading speed of the participants is much greater than that of the Chinese reading speed. Chinese and English have different language characteristics, one is ideographic characters, and the other is pinyin characters, which indicates that the effect of space factor will be affected by the language characteristics. This is similar to the results of previous studies. Another reason is that for the participants, the Chinese 
sentences are compiled in their mother tongue, so the reading speed is relatively faster, while the English sentences are written in the second language, so it is more difficult for them to read. Regarding the three levels of segmentation, whether it is in Chinese or English, the reading speed of spaces between words is faster than that between characters (letters)., it can use Li, Rayner and Cave model of Chinese vocabulary to explain [23], "Chinese word recognition model think segmentation and recognition is already available in the readers mind vocabulary characterization of top-down processing and the characters of some visual characteristics caused by the interactions of bottom-up processing as a result, the glyph of visual features activated the word level characterization and transmitted to the word, and then activate the corresponding word representation" [24]. And the participants read Spaces between words need to activate the word level characterization, transmit the corresponding word to word level after activation characterization, the middle one more process, reading Spaces between words is a bottom-up processing, the differences in the reading response of the participants under the two segmentation levels of English sentences can be explained by the same principle. Therefore, the reading of sentences with spaces between words will be faster than sentences with spaces between characters.

\section{CONCLUSION}

In this study, the researchers found that regardless of Chinese or English reading materials, word space text presented a faster reading time for conditional sentences; words were an important processing unit. The reading speed of the spaces between words was obviously faster than the other two conditions, and the spaces played a great role in promoting the vocabulary recognition of pinyin characters. When the reading material was in Chinese, the three segmentation methods will not have much impact on the participant's reading; while when the reading material was in English, there was a significant difference in the reading rate of the participants between the spaces between letters, spaces between words, and spaces between non-words.

\section{AUTHORS' CONTRIBUTIONS}

Dixiu Liu and Yanping Ling conceived and supervised the experiments; Dixiu Liu and Yanping Ling designed the experiments; Yanping Ling implemented the experiments and collected data; Yanping Ling analyzed the results; Dixiu Liu and Yanping Ling wrote and revised the paper.

\section{ACKNOWLEDGMENTS}

This research was supported by grants from the Social Science Project of Ganzhou City (Grant 19468 to Dixiu Liu).

\section{REFERENCES}

[1] Wang, D. (2011). The influence of word segmentation on Chinese reading of Russian students. Tianjin Normal University.

[2] Xie, H. Y. (2006:1-57). Research on the Chinese Word Boundary Parsing Ability of Intermediate and Advanced Foreign Students. GuangDong: Jinan University.

[3] Li, X. S., Liu, P. P., Ma, G. J. (2011). Effects of Word Segmentation on Cognitive Mechanisms During Chinese Reading. Advances in Psychological Science, 19(04) : 459-470.

[4] Li, Y. Y. (2019). A Correlational Study Between Word Segmentation and Reading Ability of CFL Learners at Intermediate and Advanced Stages in Non-target Language Environment. East China Normal University.

[5] Sun, F., Morita, M, \&Stark, L.W. (1985). Comparative patterns of reading eye movement in Chinese and English. Perception\& Psychophysics, 1985,37 (6) :502506.

[6] Winskel,H., Radach, R., \& Luksanneeyanawin, S. (2009). Eye movements when reading spaced and unspaced Thai and English: A Comparison of Thai - English Bilinguals and English Monolinguals. Journal of Memory and Language. 61:339-351.

[7] Li, X., Bai, X.J.,Yan, G.L., Liang, F.F. (2010). The Role of Space During Reading. Advances in Psychological Science, 18(121): 21-29.

[8] Bai, X. J., Tian, J., Yan, G.L., Wang, T. L. (2009). Effect of word segmentation on Chinese text reading: Evidence from American Students' Eye Movements. Nankai Journal of Linguistics, (01): 140-153+184-185.

[9] Shen, D. L., Liversedge, S. P., Tian, J., Zang, C. L., Cui, L., Bai, X. J., Rayner, K. 
(2012).Eye movements of second language learners when reading spaced and unspaced Chinese text $[\mathrm{J}]$. Journal of Experimental Psychology: Applied, 18(2): 192-202.

[10] Yu, X. L., Ren, Z. H., Ye, Y. D. (2015). The Effects of Interword Spacing in Chinese Text Reading:A Meta-analysis Based on Eye Movements Research. Advances in Psychological Science, 23(183): 30-45.

[11] Bai, X. J., Guo, Z.Y., Cao, Y. X., Gu, J.J, Yan, G.L. (2012). Effect of word segmentation on older adults: evidence from eye movements. Chinese Journal of Gerontology, 32: 120-122.

[12] Liu, I. M., Yeh, J. S., Wang, L. H., \& Chang, Y. K. (1974). Effects of arranging Chinese words as units on reading efficiency. Chinese Journal of Psychology, 16, 25-32.

[13] Tian, J. (2009). The effect of word segmentation on Chinese text reading: Evidence from Korean and Thai Students' Eye movements. Tianjin Normal University.

[14] Bai, X., G. Yan and C. Zang. (2008) Reading spaced and unspaced Chinese text: evidence from eye movements $[\mathrm{J}]$. Journal of Experimental Psychology Human Perception \& Performance, 34(5): 1277.

[15] Gao, S. (2006). The Eeffet of Word Boundaries on Reading for Learners of Chinese as a Seeond Language. Beijing: Beijing Language and Culture University, 160.

[16] Bai, X.J., Guo, Z.Y., Gu, J.J., Yan, G.L., Zang, C.L. (2012). Effects of Word Segmentation on hindi-English Bilinguals' Reading: Evidence from Eye Movements. Journal of Psychological Science, 35 (197): 34-39.

[17] Perea, M., \& Acha, J. (2009). Space information is important for reading. Vision Research, 49:1994-2000.

[18] Spragins A B, Lefton L A, Fisher D F. (1976). Searching spatially transformed text of Eye move-ments while reading: A developmental examination. Memory and Cog-nition. 4: 3642.

[19] Rayner K, Pollatsek A. (1996). Reading unspaced text is not easy: Comments on the Implications of Epelboim's study for models of eye movement control in Reading.Vision Research. 36: 461-465.

[20] Morris R K, Rayner K, Pollatsek A. (1990). Eye movement guidance in reading. Journal of Experimental psycho-ogy: Human Perception and Performance. 28: 1107-1118.

[21] Malt B C, Seamon J G. (1978). Peripheral and cognitive com-ponents of eye guidance in FillSpace Reading. Per-ception \& Psychology. 23: 399-402.

[22] Hsu, S-H.,\& Huang, K.C.(2000). Effects of word-spacing on reading Chinese text from a video display terminal $[\mathrm{J}]$. Perceptual and Motor Skill. 90: 81-92.

[23] Rayner, K., Fischer, M.H.,\& Pollatsek, A. Unspaced text interferes with both word identification and eye movement control, Vision Research, 1998,38(8): 1129-1144.

[24] Zeng, Y. X. (2017). Infant Word Segmentation: The Current Situations and the Expectation. The Science Education Article Collects, No.374, 165-166. 